\title{
Study of febrile thrombocytopenia in Malwa region of India
}

\author{
Vishal Yadav', Abhishek Singhai ${ }^{2}$ \\ ${ }^{1}$ Assistant Professor, Department of Medicine, Sri Aurobindo Medical College \& PGI, Indore, India, ${ }^{2}$ Assistant \\ Professor, Department of General Medicine, All India Institute of Medical Sciences, Bhopal, India
}

Background: Febrile thrombocytopenia is the thrombocytopenia associated with fever. Diseases which commonly present with fever and thrombocytopenia are malaria, leptospirosis, septicemia, typhoid, arbovirus diseases such as dengue. Aims and Objectives: The study was intended to know the underlying etiology of febrile thrombocytopenia in Malwa region and its various presentations. Materials and Methods: This study was conducted at Department of General Medicine of a tertiary care centre for two years. Five-hundred adult patients (age $>18$ years) of febrile thrombocytopenia were evaluated for possible inclusion in this study. Routine investigations including complete blood counts, peripheral smear examination, kidney function tests, liver function tests, ultrasound of abdomen were done in all subjects. The specific investigations were done as and when indicated. Results: Out of 500 patients of febrile thrombocytopenia, 298 were male, while 202 were female. Commonest symptom after fever was malaise (83\%) followed by nausea-vomiting (76\%), headache $(63.4 \%)$, jaundice $(26.8 \%)$, easy fatigability $(22.4 \%)$, bleeding manifestations $(7.8 \%)$, cough $(6.8 \%)$, dyspnea $(5.8 \%)$, rash $(2.4 \%)$ and delirium (1.4\%). Dengue was found in 178 patients. Malaria was found in 122 patients. Other causes of febrile thrombocytopenia were septicemia (22 cases), leptospirosis (12 cases), chikungunya fever (10 cases), viral hepatitis (9 cases), enteric fever (9 cases), acute lymphoblastic leukemia (2 cases), non-hodgkin lymphoma (one case), disseminated tuberculosis (one case). Conclusion: Febrile thrombocytopenia is an important clinical condition commonly caused by infections, particularly viruses and malaria. Such studies will help in finding changing trends of locally prevalent infectious diseases and finding some new emerging diseases not prevalent in the particular region.

Access this article online

Website:

http://nepjol.info/index.php/AJMS

DOI: 10.3126/ajms.v8i5.17633

E-ISSN: 2091-0576

P-ISSN: $2467-9100$

Key words: Fever, Thrombocytopenia, Malwa, Infection, Malaria, Dengue

\section{INTRODUCTION}

Thrombocytopenia is defined as a reduction in the peripheral blood platelet count below the lower normal limit of $150000 / \mu \mathrm{L}$. Thrombocytopenia occurs due to decreased platelet production, which occurs in conditions such as vitamin B12 and folate deficiency, leukemia, sepsis (bacterial or viral infection) and hereditary disease. Thrombocytopenia may also occur due to increased destruction such as idiopathic thrombocytopenic purpura, thrombotic thrombocytopenic purpura (TTP), hemolytic uremic syndrome (HUS), disseminated intravascular coagulation, paroxysmal nocturnal hemoglobinuria, systemic lupus erythematosus, antiphospholipid syndrome, post-transfusion purpura and hypersplenism. Drugs, which can cause thrombocytopenia, are quinine, valproic acid, methotrexate, carboplatin, interferon, isotretinoin and heparin. ${ }^{1}$ Febrile thrombocytopenia is the thrombocytopenia associated with fever. Diseases which commonly present with fever and thrombocytopenia are malaria, leptospirosis, rickettsial infections, septicemia, typhoid, borreliosis, arbovirus diseases such as dengue or yellow fever, rodent-borne viruses such as Hanta and Lassa fever, human immunodeficiency virus, visceral leishmaniasis and TTP-HUS. ${ }^{2}$ The study was intended to know the underlying etiology of febrile thrombocytopenia in malwa region and its various presentations. 


\section{MATERIALS AND METHODS}

This is an observational study, conducted at Department of General Medicine of a tertiary care centre. The duration of study was two years; November-2014 to October-2016.Purposive sampling technique is used for selection of desired samples according to inclusion criterion. 500 adult patients (age $>18$ years) of febrile thrombocytopenia were evaluated for possible inclusion in this study. All adults with platelet count $<150000 / \mu \mathrm{L}$ with fever were included in this study. The exclusion criteria were patients of thrombocytopenia without fever, diagnosed cases of thrombocytopenic purpura on treatment, patients on chemotherapy, patients on treatment with antiplatelet and other drugs causing thrombocytopenia, patients with cirrhosis of liver, portal hypertension, connective tissue disorder like systemic lupus erythematosus.

Demographic characters like age, sex, height, weight of all subjects were noted. Detail history was recorded, general physical examination was done and detailed systemic examination was done. Routine investigations including complete blood counts, peripheral smear examination, kidney function tests, liver function tests, ultrasound of abdomen were done in all subjects. The specific investigations were done as and when indicated. Both thick and thin blood smears were examined for malarial parasites as well as for, platelet count, its morphology and for any abnormal cells. Special investigations like NS1 antigen for dengue, IgM Elisa for dengue, malarial antigen, Elisa for HIV, HbsAg, IgM Elisa for leptospira, blood culture and sensitivity, body fluid analysis and bone marrow aspiration were done as and when needed. Diagnosis of Viral fever was made whose definitive diagnosis was not made after specific investigations and responded to symptomatic treatment.

Ethical Consideration: Prior to conduct of the present study, the protocol of the study was submitted to ethical and scientific committee of hospital. After getting due approval from these two committees, the present study was initiated. Also prior to conduct of study related procedure/investigation, a voluntary written informed consent was taken from the patient/legally acceptable representative.

Statistical Technique: Microsoft Excel ${ }^{\circledR}$ and SPSS ${ }^{\circledR} 20$ for Windows ${ }^{\circledR}$ were used for data storage and analysis. The qualitative data were expressed in percentages and quantitative data were expressed as mean \pm standard deviation. Student's t test and Chi-Square test were used to determine statistical difference between variables.

\section{RESULTS}

Out of 500 patients of febrile thrombocytopenia, 298 (59.6\%) were male, while 202 (41.4\%) were female. All subjects were in age group 18-68 years with mean age $( \pm$ SD) 39.6 years $( \pm 21.1) .242$ patients $(48.4 \%)$ had only thrombocytopenia, 140 patients $(28 \%)$ had thrombocytopenia and anemia, 68 patients $(13.6 \%)$ had thrombocytopenia and leucopenia, and 48 patients $(9.6 \%)$ had pancytopenia. Commonest symptom after fever was malaise $(83 \%)$ followed by nausea-vomiting $(76 \%)$, headache $(63.4 \%)$, jaundice $(26.8 \%)$, easy fatigability (22.4\%), bleeding manifestations $(7.8 \%)$, cough $(6.8 \%)$, dyspnea (5.8\%), rash (2.4\%) and delirium (1.4\%) (Table 1).

Dengue was found in 178 patients among them 98 patients were NS1 antigen positive while 80 patients were positive for both NS1 and Ig M antibody. Malaria was found in 122 patients in which $48 \%$ patients had Plasmodium falciparum infection followed by Plasmodium vivax in $42 \%$ and mixed infection in $2 \%$. Viral fever was seen in 178 patients. Other causes of febrile thrombocytopenia were septicemia (22 cases), leptospirosis (12 cases), chikungunya fever (10 cases), viral hepatitis (9 cases), enteric fever (9 cases), acute lymphoblastic leukemia ( 2 cases), non-hodgkin lymphoma (one case), disseminated tuberculosis (one case) (Table 2).

\begin{tabular}{lc}
$\begin{array}{l}\text { Table 1: Presenting signs/symptoms of cases of } \\
\text { febrile thrombocytopenia }\end{array}$ \\
\hline Clinical signs/symptoms & Number of cases (\%) \\
\hline Fever & $500(100)$ \\
Malaise & $415(83)$ \\
Vomiting/nausea & $380(76)$ \\
Headache & $288(63.4)$ \\
Jaundice & $134(26.8)$ \\
Easy fatigability & $112(22.4)$ \\
Bleeding manifestations & $39(7.8)$ \\
Cough & $34(6.8)$ \\
Dyspnea & $29(5.8)$ \\
Rash over body & $12(2.4)$ \\
Delirium & $7(1.4)$ \\
\hline
\end{tabular}

\begin{tabular}{lc} 
Table 2: Causes of febrile thrombocytopenia \\
\hline Cause & Number of cases (\%) \\
\hline Viral fever & $178(35.6)$ \\
Dengue fever & $134(26.8)$ \\
Malaria & $122(24.4)$ \\
Septicemia & $22(4.4)$ \\
Leptospirosis & $12(2.4)$ \\
Chikungunya fever & $10(2)$ \\
Viral hepatitis & $09(1.8)$ \\
Enteric fever & $09(1.8)$ \\
Hematological malignancy & $03(0.6)$ \\
Disseminated tuberculosis & $01(0.2)$ \\
\hline
\end{tabular}


In our study, 59 patients $(11.8 \%)$ had platelet count $<20,000 / \mu \mathrm{L}$, followed by 69 patients $(13.8 \%)$ in the range of $20,000-50,000 / \mu \mathrm{L}, 269$ patients $(53.8 \%)$ in the range of $50,001-1,00,000 / \mu \mathrm{L}$ and 103 patients $(20.6 \%)$ had $>$ $1,00,000$ but $<1,50,000 / \mu \mathrm{L}$ (Table 3).

\section{DISCUSSION}

Febrile thrombocytopenia is commonly encountered problem especially during monsoon and Pre- monsoon period. Infections with protozoa, bacteria and viruses can cause thrombocytopenia with or without disseminated intravascular coagulation. Infections cause decrease in platelet count both due to effects on platelet production and platelet survival. Thrombocytopenia in bacterial infections can occur as a part of sepsis with disseminated intravascular coagulation. Patients with sepsis may also develop hemophagocytic histiocytosis with phagocytosis of platelets and leucocytes in the bone marrow histiocytes. Both Gram-positive and Gram-negative bacterial infections can lead to sepsis. Elevated platelet-associated IgG has been implicated. Platelets tend to adhere to damaged vascular surfaces in meningococcemia.

Viruses produce thrombocytopenia by various mechanisms like impaired platelet production as a result of direct viral invasion, toxic effect of viral proteins on thrombopoiesis, virus induced hemophagocytosis and increased platelet destruction caused by binding of virus-induced autoantibodies or viral antigen antibody complexes. Thrombocytopenia in dengue infection raises concern about bleeding risk. Bone marrow suppression by virus and peripheral destruction of platelets has been implicated. Platelet transfusions are not routinely recommended in the management of Dengue fever. ${ }^{3}$ According to recent guidelines by the World health organization and National Vector-borne Diseases Control Programme prophylactic transfusion of platelets is not indicated unless the patient has bleeding or a count of less than $10000 / \mu \mathrm{L} .{ }^{4}$ Thrombocytopenia during malarial infection may appear even before fever, anemia and splenomegaly become manifest. ${ }^{5}$ Immune mediated lysis, sequestration in the spleen and a dyspoietic process in marrow with diminished platelet production have all been postulated. During early stages of malaria, platelet agglutination

\begin{tabular}{|c|c|}
\hline Platelet count & Number of cases (\%) \\
\hline$<20000 / \mu \mathrm{L}$ & $59(11.8)$ \\
\hline $20000-50000 / \mu \mathrm{L}$ & 69 (13.8) \\
\hline $50001-100000 / \mu \mathrm{L}$ & $269(53.8)$ \\
\hline$>100000-<150000 / \mu \mathrm{L}$ & $103(20.6)$ \\
\hline
\end{tabular}

as a result of endothelial cell activation and release of activated von-Willebrand factor occurs which may cause thrombocytopenia. ${ }^{6}$ Occasionally platelets can be invaded by malarial parasites. Thrombocytopenia in malaria is rarely severe and treatment is focused on eradication of malarial parasite.

A total of 500 cases of febrile thrombocytopenia were studied with 298 males and 202 females. The commonest etiology of febrile thrombocytopenia in this study was viral fever $(35.6 \%)$. The other etiologies were dengue fever (26.8\%), malaria (24.4\%), septicemia (4.4\%), leptospirosis $(2.4 \%)$, chikungunya fever $(2 \%)$. In a study by Kumar P et al, ${ }^{7}$ malaria was the commonest etiology. In a similar study by Nair PS et al, ${ }^{8}$ they found septicemia as the commonest cause for febrile thrombocytopenia. A study by Gandhi $\mathrm{AA}$ et $\mathrm{al}^{9}$ also found dengue and malaria as the common causes of febrile thrombocytopenia. In the present study population, malaise ( $83 \%$ ) was the most common clinical feature after fever. Similar result were obtained in study by Tong Seng Fahet et $\mathrm{al}^{10}$ i.e. myalgia being the commonest clinical feature. In the present study, bleeding manifestation was present in $39(7.8 \%)$ cases. In the study by Nair PS et al, ${ }^{8}$ bleeding manifestation observed in $41.3 \%$ cases. In another study by Kumar P et al, ${ }^{7}$ bleeding manifestation seen in $11.05 \%$ cases. In the present study most common platelet distribution 50,001-1, 00,000/cumm was seen in $53.8 \%$ cases. Similar results $(56.8 \%)$ were obtained in Nair PS et $\mathrm{al}^{8}$ study for this platelet distribution range. Severe thrombocytopenia, i.e.count $<20,000 /$ cumm observed in $11.8 \%$ cases in present study as compared to $17.4 \%$ cases in study by Nair PS et al ${ }^{8}$ and $13.39 \%$ cases in study by Gandhi AA et al. ${ }^{9}$

\section{CONCLUSION}

Febrile thrombocytopenia is an important clinical condition commonly caused by infections, particularly viruses and malaria. It commonly manifests as symptom/signs of underlying condition and sometime with bleeding manifestation. Treatment of underlying condition will lead to rapid improvement in platelet count with complete clinical recovery. Such studies will help in finding changing trends of locally prevalent infectious diseases and finding some new emerging diseases not prevalent in the particular region.

\section{REFERENCES}

1. Bichile SK. Platelet disorder. API Textbook of Medicine. Vol. 1, $9^{\text {th }}$ edition, Munjal YP (Ed.), Jaypee Brothers: New Delhi 2012; 987-988.

2. Lee GR, Foerster J, Lukens J, Paraskevas F, Greer JP, Rodgers GM. Shirley Parker Levine - Miscellaneous causes of 
thrombocytopenia. In: Wintrobe's Clinical Haematology. Vol. 2, $10^{\text {th }}$ edition, Lipincott Williams: Philadelphia 1999; 1623-1629.

3. Ganesan N, Gunasekharan I and Padhi S. Platelet phagocytosis in peripheral blood during acute phase of dengue virus infection. J Curr Res Sci Med 2015; 1:51-53.

4. Lye DC, Lee VJ, Sun Y and Leo YS. Lack of efficacy of prophylactic platelet transfusion for severe thrombocytopenia in adults with acute uncomplicated dengue infection. Clin Inf Dis 2009; 48:1262-1265.

5. Jadhav UM, Patkar VS and Kadam NN. Thrombocytopenia in malaria-correlation with severity and type of malaria. J Assoc Physicians India 2004; 52:615-618.

6. De Mast Q, Groof E, Lenting PJ, de Groot PG, McCall M, Sauerwein RW, et al. Thrombocytopenia and release of activated von Willebrand factor during early malaria. J Inf Dis 2007; 196:622-628.

7. Kumar $P$ and Chandra KA. Clinical Study of Febrile Thrombocytopenia: A Hospital based retrospective study. Indian Journal of Clinical Practice 2014; 24:952-957.

8. Nair PS, Jain A, Khanduri U and Kumar V. A Study of fever associated with thrombocytopenia. JAPI 2003; 51:1173.

9. Gandhi AA and Akholkar PJ. Clinical and Laboratory Evaluation of Patients with Febrile Thrombocytopenia. NJMR 2015; 5:43-46.

10. Tong SengFah, Noorazah Abdul Aziz, Chin GekLiew and Khairani Omar. Clinical features of Acute Febrile Thrombocytopenia among Patients attending primary Care Clinics. Malaysian Family Physician J 2006; 1:16-18.

\section{Authors Contribution:}

VY-Concept and design of the study, manuscript preparation; AS- Concept and design of the study, manuscript preparation, statistically analyzed and interpreted, critical revision of the study, review of literature, helped in preparing the first draft and collecting data

Work attributed to:

Department of Medicine, Sri Aurobindo Medical College \& PGI, Indore, India

Orcid ID:

Dr. Vishal Yadav: (D) http://orcid.org/0000-0003-1506-8991

Dr. Abhishek Singhai: (i) http://orcid.org/0000-0002-3216-3387

Source of Support: None, Conflict of Interest: None declared. 